\title{
Proactive decremental effects on response speed in a continuous DRT task
}

DIga Favreau

MCGILL UNIVERSITY

\begin{abstract}
Thirty Ss performed a 324-trial, subject-paced, twosignal, frequency-imbalanced, disjunctive reaction time task. Responses on trials following the occurrence of the infrequent signal yielded longer RTs than responses on other trials. This effect is attributed to novelty produced "startle," and is different from series effects previously reported.
\end{abstract}

\section{Introduetion}

In a disjunctive reaction time (DRT) task one signal is presented on each trial, each class of signals ( $\mathrm{Sa}$, $\mathrm{Sb}$, etc.) requiring a different response ( $\mathrm{Ra}, \mathrm{Rb}$, etc.). Some interest has recently been shown in the relation of DRT to the characteristics of the series in which individual trials occur. In such studies the latency of response (RT) to each signal class has been found to be a function of its probability of occurrence in the series, the shorter latencies accompanying responses to the more probable signal classes (e.g., Fitts et al., 1963). In the course of a study (Favreau, 1964) of the effects on RT of stimulus variation within signal classes, this author discovered a proactive effect which was associated with both signal class probability and stimulus variation within signal classes. The task allowed the subject (S) to pace the presentation of signals himself, and it was in the latency of this pacing response that the main effect occurred. The part of the study that demonstrates this effect, and bears on its interpretation, is reported here.

\section{Method}

Transparaent images of eight different geometric forms in each of five colors served as signals. Each signal had only one shape and one color. One of these was projected briefly $(0.2$ sec.) on each trial. S was instructed to operate one of a pair of keys if the projected figure was a circle, and to press the other key if the figure was any other shape. Three seconds after S's response to the signal, a ready signal (jewel light) appeared. Now $\mathrm{S}$ had to press another key to cause the next signal to be projected; this is called the pacing or starting response. The signal class "circles" (Sa) was presented with $1 / 8$ the frequency of the signal class "non-circles", (Sb). The experimental session consisted of 324 trials.

Thirty men were divided into three equal groups. Each group was tested under one of three experimental treatments, representing different degrees of stimulus variation. These differences were introduced by varying the shape or color, or both, of the signals. Thus, in the condition labeled No Change (NC) all $\mathrm{Sb}$ signals

\begin{tabular}{|c|c|c|c|c|c|c|c|c|}
\hline \multirow[t]{2}{*}{ Condition } & Means & $(\sec )$. & $\mathrm{t}$ & $\mathrm{p}$ & Means & $(\mathrm{sec})$. & $\mathrm{t}$ & $\mathrm{p}$ \\
\hline & $\mathrm{STa}$ & STb & & & $\mathrm{RTb}, \mathrm{a}$ & $\mathrm{RTb}, \mathrm{b}$ & & \\
\hline $\mathrm{CC}$ & 1.24 & 1.15 & $2.44<$ & $<.05$ & .62 & .60 & 1.01 & NS \\
\hline IC & 1.32 & 1.18 & $2.80<$ & .05 & .72 & .71 & .80 & NS \\
\hline NC & 1.14 & 1.06 & $2.80<$ & $<.05$ & .71 & .68 & 3.57 & 6 .01 \\
\hline
\end{tabular}

were blue squares, Sa signals (circles) were also blue. In the condition called Intermittent Change (IC) Sb signals were any of three shapes and five colors; the same $\mathrm{Sb}$ signal could occur as frequently as five times in succession. In the Constant Change condition (CC) $\mathrm{Sb}$ signals were any of eight shapes and five colors; no signal of the same shape or color was allowed to occur on two successive trials. In the last two conditions the Sa signals (circles) could appear in any color. In all three conditions, the Sa signals occurred on the same trials in the series.

Four measures of response and pacing speed were taken:

RTa: the interval between the onset of the infrequent signal $\mathrm{Sa}$ (circle) and the response, Ra;

$\mathrm{RTb}$ : the interval between the onset of the frequent signal $\mathrm{Sb}$ (any non-circle) and the response, $\mathrm{Rb}$;

STa: the interval between the onset of the ready light and the pacing response when the previous signal had been $\mathrm{Sa}$; this will be referred to as STa (read: starting time after Sa);

STb: the interval between the onset of the ready light and the pacing response when the previous signal had been Sb; this will be referred to as STb (read: starting time after $\mathrm{Sb}$ ).

\section{Results}

As expected (see Introduction), RTa was significantly longer than RTb. The unexpected finding-the one of interest here-was that, under all three conditions, STa was significantly longer than STb (see Table 1). That is, responding to the less probable signal (Sa) was followed by an increase in the time taken to initiate (pace) the following trial. Analysis of this proactive effect was carried one step further by obtaining the mean of RTb for the trial immediately following each Ra $(\mathrm{RTb}, \mathrm{a})$ and 
comparing it with the mean latency of all other $\mathrm{Rb}$ trials combined $(\mathrm{RTb}, \mathrm{b})$. As seen in the right half of Table 1 , whereas there were no significant differences in the CC and IC conditions, in the case of the NC condition, $\mathrm{RTb}$, a was significantly longer than $\mathrm{RTb}, \mathrm{b}$.

\section{Discussion}

The results of the present experiment indicate that there is a decrease in response speed following the execution of a discriminative response to a less probable signal class. This is clearly seen in the speed of initiating the next pacing response, but may also extend to the next discriminative response, as the difference between RTb, a and RTb, b in the NC condition indicates. A hypothesis that may profitably be applied to these results is that the occurrence of a less probable signal startles $\mathrm{S}$, and that the startle reaction disrupts his performance for a few (at least three) seconds. Evidence for the disruptive effects of startle associated with sudden stimulus change or novelty comes from studies of both human task performance (e.g., Maheux, 1964) and animal learning (e.g., Claus \& Bindra, 1960). Note that in this study the effect cannot be attributed wholly to stimulus change, since STa was longer than STb even in the $\mathrm{CC}$ condition, which involved stimulus change on every trial. Therefore, the postulated startle effect must be related, at least in part, specifically to the novelty of the less probable signal class (Sa) and/or to the necessity of making the corresponding, less probable, response ( $\mathrm{Ra})$. That stimulus change alone can enhance the startle effect can be seen in the prolonged speed decrement under the NC condition. In this condi- tion, Sa was preceded by an unvarying pattern of stimulation, and it is known that the longer the experience $\mathrm{S}$ has with an unvarying stimulation, the greater is the novelty provided by any given change in it (e.g., Bindra, 1959; Bindra \& Seely, 1959).

The above findings suggest that the role of noveltystartle in other series effects observed in DRT experiments should be investigated.

\section{References}

BINDRA, D. Stimulus change, reactions to novelty, and response decrement. Psychol. Rev., 1959, 66, 96-103.

BINDRA, D., \& SEELY, J. F. Response decrement as a function of amount of training. J.exp. Psychol., 1959, $57,317-322$.

CLAUS, H. J., \& BINDRA, D. Reactions to novelty and stimulus-change induced response decrement. Canad. J. Psychol., 1960, 14, 101-110.

FAVREAU, O. Stimulus variation and the speeds of reaction and pacing. Unpublished master's thesis, McGill University, 1964.

FITTS, P. M., PETERSON, J. J., \& WOLPE, G. Cognitive aspects of information processing: II. Adjustments to stimulus redundancy. J.exp. Psychol., 1963, $65,423-432$.

MAHEUX, M. Distraction effects and stimulus generalization. Psychol. Rec., 1964, 14, 101-112.

\section{Note}

1. The research reported in this paper was supported by a grant (No. 9425-10) from the Defence Research Board of Canada to Dr. Dalbir Bindra. 